\title{
Medical Allocations to Persons with Special Needs during a Bioterrorism Event
}

\author{
Donald E Brannen ${ }^{1}$, Melissa Branum ${ }^{1}$, Sejal Pawani ${ }^{1}$, Sandy Miller ${ }^{2}$, Jeanne Bowman ${ }^{3}$, and \\ Tracy Clare 4
}

1. Greene County Public Health, Greene County, Ohio

2. Clark County Combined Health District

3. Champaign County Health Department

4. Dayton-Montgomery County Public Health

\begin{abstract}
After the bioterrorism-anthrax attacks of 2001, public health officials were tasked with planning population-wide medicine dispensing. This planning started with assumptions and then evaluations of seasonal immunization clinics. Research on the 2009 H1N1 pandemic-vaccination campaign showed that an adequately prepared public health system could have prevented over $16 \%$ of fluassociated hospitalizations. The $\mathbf{2 0 1 1}$ ice storms revealed difficulties with sheltering medically fragile persons with disabilities. Later research showed that training and preparedness levels increased responders' willingness to serve. When triaging disaster survivors to community-mass-care-services of general shelters, medical shelters, or mental health services; sorting improved up to $15 \%$ when past traumatic effects, personal care assistance, or service methodology were accounted for. The number of persons who are disabled and dependent on electric medical equipment are increasing. This current study compared the time it takes to dispense medication to two different cohorts: a general-population cohort $(n=31)$ and a special-needs cohort $(n=30)$. The cohort comprised entirely of persons with special needs took 4.1 compared to 2.48 minutes per person in a general population cohort $(p=.057)$. A person with any special needs took 3.73 versus 2.43 minutes for a person with no special needs $(p=.082)$. Modeling of service times per station and cohort type found significant delays at the medical station among persons in the general population who are pregnant (14 minutes or 840 seconds, $p=.002$ ) and persons in the special needs cohort with a language barrier (12.5 minutes or 750 seconds, $p=.001$ ). Recommendations include planning for closed Points of Dispensing Sites (PODS) to those with special needs, ensuring a sufficient number of medical dispenser in open PODS, and assigning extra capacity at the medical station area for special needs involving children, language, or pregnancy issues.
\end{abstract}

Correspondence: Dr. Donald E Brannen, Greene County Public Health, Greene County, Ohio, Xenia, 45385 OH, USA+1 937 374-5600. dbrannen@gcph.info

DOI: 10.5210/ojphi.v8i3.6977

Copyright (C2016 the author(s)

This is an Open Access article. Authors own copyright of their articles appearing in the Online Journal of Public Health Informatics. Readers may copy articles without permission of the copyright owner(s), as long as the author and OJPHI are acknowledged in the copy and the copy is used for educational, not-for-profit purposes. 


\section{Introduction}

Even before the bioterrorism attacks of 2001, there had been concern about the capacity of local public health to protect the public from an anthrax attack. Strategies to get lifesaving medicine to those exposed were among the critical-bioterrorism-preparedness issues identified after the post 9/11 anthrax attacks in 2001 [1]. In Ohio, like many other states, there are standing medical orders for the prophylactic use of antibiotics and vaccination in response to a release of a bioterrorism agent like smallpox or anthrax. These orders do not cover persons with known disease rather only those persons with suspected exposures. If the anthrax vaccine was made available under an Emergency Use Authorization and the Centers for Disease Control and Prevention released the vaccine to the area with suspected exposure to the bioterrorism agent Bacillus anthracis, public health staff, augmented with Medical Reserve Corps (MRC) volunteers would dispense the vaccine, along with antibiotics. Dispensing post-exposure prophylaxis medications to a large number of people at a well-designed clinic after a release of anthrax spores, results in more medicine dispensed per unit time, allowing for fewer deaths and faster epidemic eradication [2-4]. If local public health is unable to adequately respond to an anthrax attack, anthrax can cause illness or death 1 to 6 days after exposure.

After the 2001 anthrax attacks, concurrent with preparation for a potential smallpox attack, individual public health officials had to make broadly applicable assumptions about staffing a mass clinic [5]. In the following years, local public health agencies started to evaluate their ability to conduct mass immunization clinics. These evaluations included studies of the total number of persons served within a specific time and the amount of resources required. Nine county health departments in the West Central Region of Ohio conducted studies of the region's seasonal immunization clinics during the 2005-2006 flu season; with over 7,000 vaccinated and 997 randomly selected observations. The effectiveness at providing the required medical coverage to protect the population was associated with how the dispensing site was setup [6]. The clinic designs were one of three styles: traditional, mass, or queue designs. The traditional setup comprised serialaligned single stations, one person at a time per station. The queue setup comprised each station having a single waiting line. The mass setup had clients arrive at multiple stations in bulk with the first open station providing service. The mass style of clinic was most effective, but the least efficient in the short term; with the number of persons served per unit time dependent on the number of vaccinators. The number of lives saved by mitigating disease spread would allow the cost efficiency of the queue and mass styles to exceed the traditional clinic design with increasing virulence of the bioagent. However, independent of available resources, communities can have a variable response to mitigating disease spread, just by the type of culture that is prevalent within the responding organizations [7].

The United States government has stockpiled equipment and medicine in the SNS to respond to disasters. These evaluations evolve around the Strategic National Stockpile (SNS), a cache of medicine and supplies that would be used to mitigate the health effects from a terrorist attack with a chemical, biological or nuclear weapon [8]. There is concern on how the supplies would be deployed in response to a disaster when the number of patients exceeds the ability of the health care system to respond [9]. For example, anthrax is a bacterium that can cause illness after 1 to 6 days of exposure. One source of variability in the response is having too many people show up at any particular time. Too many people increase the time spent in waiting lines. Any delay in service 
times can start an exponential increase of wait times for people who are just arriving for service. Having persons wait at a mass dispensing clinic increases the chance for sequela or unrelated consequences to occur like a heart attack. In a disease outbreak this can also increase the inadvertent transmission of the disease causing agent. These inadvertent and unrelated events to the primary mission of dispensing the correct medicine in the right dosage can draw off essential staff from their mission. Other issues that can arise from having too many people include increased stress and anxiety, which in turn may lead to further disruptions in the clinical dispensing process. A bad scenario is that so many people arrive that the crowd-surge itself causes injury and even worse the crowd-surge turns into a riot.

The SNS, maintained by the Centers for Disease Control and Prevention (CDC) was started in 1999 with a budget of about \$50 million [8]. The SNS inventory includes a variety of medicines and equipment to deal with public health emergencies including all sorts of natural disasters and bioterrorism events. Included in the inventory are medicines to prevent anthrax before the onset of symptoms. Immunoglobulin can treat current infection, but broad based antibiotics are the first line of defense in a population based exposure. If anthrax is suspected, there are standing orders to dispense prophylactic antibiotics and provide anthrax vaccine to persons with known or suspected exposure to Bacillus anthracis. Either doxycycline $100 \mathrm{mg}$ by mouth for 60 days or ciprofloxacin $500 \mathrm{mg}$ twice per day for 60 days is recommend in adults, with other specific recommendations for children and pregnant women (Ohio Department of Health, Standing Medical Order, Protocol for Local Health Departments: Prophylactic Use of Antibiotics and Vaccination, April 21, 2014). Some regimens could be adjusted in conjunction with anthrax immunoglobulin and vaccine with advice from CDC.

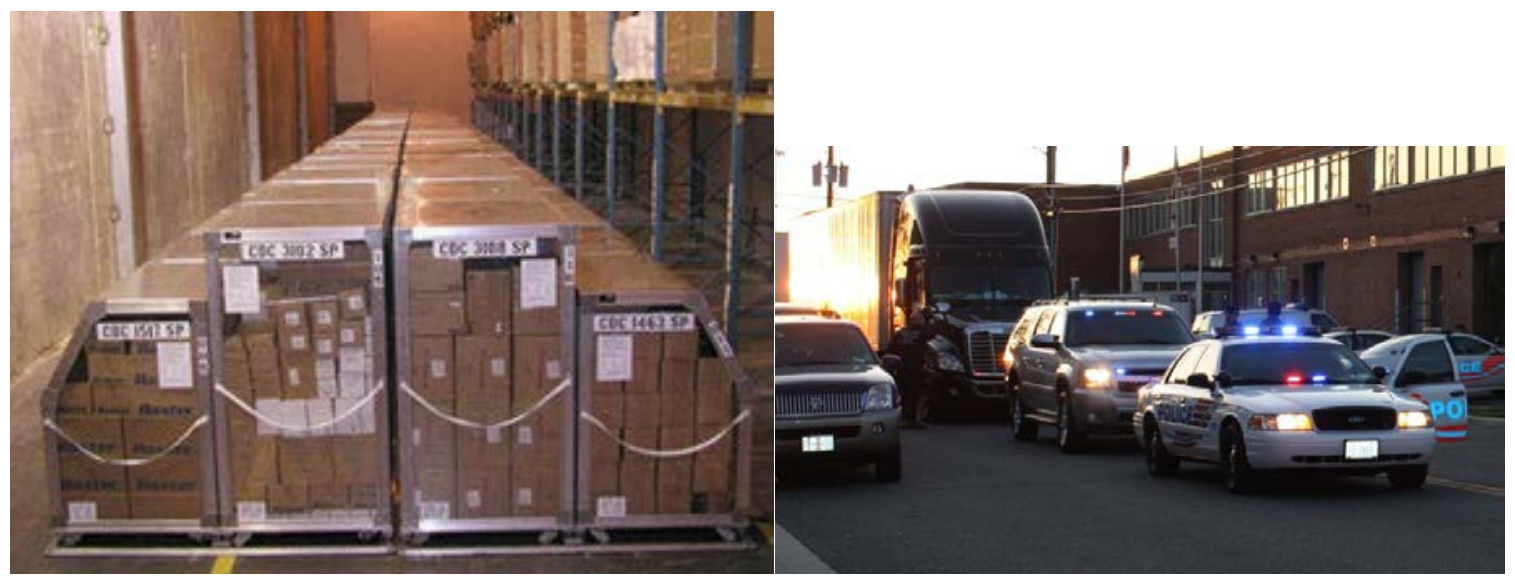

Photographs: Strategic National Stockpile supplies packaged to fit into a plane or truck for quick delivery to affected areas. (Pictures from the Centers for Disease Control and Prevention, www.cdc.gov. Downloaded 9/13/2016).

In an interview by National Public Radio’s reporter, Nell Greenfieldboyce, on June 27, 2016, it was noted that: "If there's a major anthrax attack, and there's just 48 hours to get prophylactic antibiotics to more than a million people how is it accomplished? While the federal government looks at a range of scenarios based on intelligence information and across all threats to stockpile medicine A or B, at the local level, the goal is how to get the medicines to those persons who are affected. The stockpile has 12-hour push packs with 50 tons of material to be delivered to a local 
city or town. State and local public health workers would have to figure out how to get the medicine to those who need it. Local public health officials would rely on MRC volunteers to deliver the medicines to the population. Volunteers are needed because over the last decade we have lost 50,000 state and local health officials.” [8]

Strategic National Stockpile: Cache of medicine that would be used to mitigate health effects from a terrorist attack with anthrax and other agents.

During the 2009 H1N1 pandemic, local public health was charged with providing immunizations to the population to limit illness and deaths from a new strain of influenza. To increase the effectiveness of the immunization campaign, partly due to the limited supply and capacity, persons most at risk were targeted to receive the vaccine. Once CDC delivers the stockpile, it becomes a state asset. There have been concerns that local public health was outdated, inefficient, unresponsive, and unprepared for health hazards [10]. These gaps in the capacity of public health ability to respond to a bioterrorist threat, outbreaks, and weather events have persisted [11]. Even without considering gaps in public health's infrastructure, material, or personnel; modeling of managerial inefficiencies during the 2010-2011 flu season in the United States showed that one out of every six hospitalized cases could have been prevented by better management within the overall public health system [7].

Even if local public health agencies had resources, staff, and volunteers, there is another component willingness-to-respond to an anthrax event. There are similarities among radioactive contamination and the persistence of anthrax spores, as both are persistent contaminants in the environment. The ability of communities to mobilize local MRC volunteers or Citizen's Corps volunteers in a timely manner is crucial to respond to any form of disaster including anthrax or radiation related events. The concept of a community reception center is an idea where disaster evacuees are funneled through a single center and the ability of the community is matched with their needs. In the case of a persistent environmental contaminant, the effectiveness of volunteers to route survivors to the most appropriate resource is dependent on their willingness to serve. The willing volunteer's training, medical knowledge, and preparedness significantly increased the odds $(>18: 1)$ of correctly routing survivors to the right resource [12].

Using the concept of survivors arriving at a community reception center, the next question was to determine how best to triage survivors to mental health services. Public health workers and MRC volunteers were $15 \%$ more effective at correctly triaging survivors to mental health services using an algorithm that incorporated evaluation of past trauma into the triage system [13]. The focus on improving the initial sorting of survivors to needed services was driven by a need to identify those most at risk. There is a legal mandate requiring equal access under the Americans with Disabilities Act (ADA) that persons who require assistance or accommodation for functional needs in daily life, but who do not require acute medical care for stabilization, be admitted and served in general populations shelters. In January 2011, there was an ice storm that affected the nation from New Mexico to New England. The Greene County MRC unit helped staff one of six general shelters in or near their county [14]. The MRC volunteers quickly ascertained that their shelter population was mostly comprised of persons with medical needs including insulin, oxygen, adult diapering, wheel chair assistance, and intellectual developmental issues. The shelter residents were in a tenuous state of health, initially medically stable but physically fragile; many were without their 
medicines or medical equipment. There were issues of inadequate equipment for client transfers/transport, direct medical care, and outdated medicines. The ice storm sheltering illustrates an example of the issues that may arise when a residential shelter cohort turns out to have unexpected medical needs. This event highlighted a practical separation between compliant service under the ADA and persons who require medical services during a public health emergency. Chronic care triage to a general shelter, medical shelter, or medical care was evaluated accounting for functional needs support services, the presence of a Personal Care Assistant, or the personal preference for service methodology [15]. Triage process flow that considered service methodology, the presence of personal care assistance, and those that required ongoing medical services of medically stable disasters survivors before community-mass-care translated into proportional efficiency gains up to $8 \%$ in meeting chronic care health service needs in an effective, efficient, humane, and ADA-compliant service in general emergency shelters.

\section{Definitions}

Any Special Needs: Includes persons who are disabled and persons with special needs.

Closed POD (Points of Dispensing): These are non-medical dispensing sites, such as university, colleges, schools, hospitals, nursing homes, long term care or assisted living facilities, that provide medication to people who work or reside in that site. They are basically alternate dispensing modalities.

Disability: Person with physical or mental impairment that limits one or more activities of daily living and that may require special accommodations.

Functional Needs: Has unfulfilled survival needs or requires assistance related to activities of daily living (ADL), communication, and mobility, especially to maintain degrees of independence (examples include toileting, transferring, hygiene, food preparation and consumption, temperature maintenance, and obtaining safe shelter).

Medical Needs: requires skilled nursing or medical care to maintain physical or mental health and stability as compromised by medical conditions (may be chronic, acute, or exacerbated by the disaster).

Open POD: Dispensing sites that provide medication to the general public.

Special Needs: Particular things needed (regardless of where the needs derived from, whether they are social influences or factors including limited language proficiency, breastfeeding, pet-owners, elderly, children, families, religion, race/ethnicity, cultural, or geographic influence) by or provided to help people who have a condition that makes it difficult for them to do the things that other people do.

Vulnerable: Has additional needs or influences outside of conventional expectations that impact the ability to protect or serve the self and often experiences disparity. 


\section{Background}

A release of a Bacillus anthracis spores will require provision of prophylaxis to all those exposed to the agent. The main goal of mass prophylaxis is to bring people into a location where they can be given medication, such as antibiotics and vaccination. Such locations are known as point of dispensing or POD sites. There can be open or closed POD sites. An open POD is a dispensing site which provides medication to the general public. A closed POD is not open to the public and is conducted within a defined area or facility such as a university, college, school, hospital, nursing home, long term care or assisted living facility; that provide medication to people who work or reside at that site. Closed PODs expedite dispensing of medications and have the advantage of reducing the population to be treated at an open POD. Also, such sites help in reaching out to disabled and other at risk population by bringing the medicine to the agencies that specialize in servicing that population segment. As of 2010-2014, the U.S. the disabled population was 8.5\% or 27,320,599. The number of disabled in the Dayton metropolitan area (Montgomery, Greene and Miami counties in Ohio) is shown in Table 1.

Table 1. The 2010-2014 Disability Population in Dayton Metropolitan Area, Ohio, USA.

\begin{tabular}{lccc}
\hline County name & $\begin{array}{c}\text { Population } \\
\mathbf{( 2 0 1 0} \text { census) }\end{array}$ & Disability \% & $\begin{array}{c}\text { Actual number of } \\
\text { disabled people }\end{array}$ \\
\hline Champaign & 39,145 & 14.40 & 5,637 \\
\hline Montgomery county & 535,141 & $11.3 \%$ & 60,471 \\
\hline Greene county & 161,569 & $8.1 \%$ & 13,087 \\
\hline Miami county & 102,506 & $9.2 \%$ & 9,431 \\
\hline Total & 799,216 & $10.3 \%$ & 82,989 \\
\hline
\end{tabular}

Number of disabled people in Dayton city, which is part of Montgomery county, is $15.4 \%$ of 141,761 total population (2010 census) - 21,831. Persons with disabilities need to be considered while planning for providing medical countermeasures to public health emergencies. Health department officials should keep the following statistics in consideration while planning.

- There were 378,760 Ohioans ages 5 and above (or 3.8\% of the civilian noninstitutionalized population over age 5) experiencing blindness, deafness, or a severe vision or hearing impairment.

- A higher percentage- $8.5 \%$ - of the population indicated a physical disability that substantially limits one or more basic activities such as walking, climbing, etc.

- Additional results from the Census indicate that over 516,000 Ohioans (or five percent) have a mental, physical or emotional condition that makes it difficult to concentrate, remember or learn;

- While just over 268,000 (2.6\%) have a condition that makes it difficult to dress, bathe, or get around inside the home.

- Eight percent (or 653,517 individuals) of the noninstitutionalized population ages 16 and older have a mental, physical, or emotional condition that limits their ability to go outside the home alone [16]. 
For such a population, we need to consider following alternate dispensing modalities.

- Closed POD sites with healthcare entities such as nursing homes, long term care facilities, and retirement homes can be an excellent option for institutionalized population. Closed POD staff should be trained beforehand in order to handle such situations.

- Mobile medical vans: There are people who are unable to reach the POD sites because of various reasons such as unavailability of transportation, or they are physically disabled, homebound, or institutionalized. For such people, use of mobile vans in order to reach them and provide them medications is an option.

At-risk individuals include people with access and functional needs that may interfere with their ability to access or receive medical care before, during, or after a disastrous emergency. These include children, pregnant women, people with pre-existing medical conditions, advanced age, limited English skills, homelessness, physical or mental disabilities, or other physical, mental, communication, or transportation challenges.

Planned federal updates to the 2017 Health Care Preparedness and Response Capabilities are focusing on how to assess community planning for children, pregnant women, seniors, and individuals with access and functional needs, including people with disabilities and others with unique needs. Planning to provide assistance before, during, and after an emergency to these individuals is needed. Specifically, public health planners should be aware of how to provide assistance to people with disabilities and to develop or augment existing response plans for these populations. This support of the health care system to disabled persons would prevent stress on hospitals during an emergency and would allow disabled persons to remain in their residences. Further assessment in and around specific scenarios are required to assess transport needs to prophylaxis sites, designation of medical care sites for those needing further evaluation, and assessment of specific treatment and access to care needs (e.g., partnering with regional dialysis networks) to ensure integration with prophylaxis of disabled persons potentially exposed to anthrax.

For Symptomatic Individuals: A triage area will be set up directly at the entrance to the POD and will serve to immediately screen symptomatic, sick or known exposed patients before entering the Registration Station. Those patients exhibiting symptoms and patients, who were known to be exposed but are asymptomatic, are further screened and will be transported to a hospital. Transportation of patient(s) would be provided based on need. Buses, ambulances, or other forms of transportation may be necessary [17].

In 2014, the Center for Medicaid and Medicare (CMS) mandated that all County Boards of Developmental Disabilities cease adult day services by 2019 [18]. This directive puts a burden on community agencies that are providing adult daycare, as they will now have to increase the number of people they serve. The mandate in 2014 affects all the county boards of developmental disability and all the states of the nation. Since the boards of developmental disability would have a conflict of interest as both the referral source and auditor/regulator while also providing competing services, CMS mandated that the boards could only be a coordinator or facilitator of services and can no longer provide those services themselves and to get out of the business by 2019. The change in service providers could be traumatic to the lives of those adults with developmental disabilities 
who are being served by the county boards right now. The challenge for the boards is to get out of the service by 2019 and to make it a seamless transition to their clients. This means hundreds of families in each city will be impacted, as this is a traumatic life-changing event for someone who has put their most intimate needs in others' caring hands. These daily service disruptions would be greatly magnified during a disaster or terrorism event. This mandate also impacts how dispensing would occur to this vulnerable population, as locations and providers will change over the next several years as Boards transition out of service provision to coordination and facilitator of services. Specifically, agreements entered into for a closed (not open to the public) point of dispensing site that would be dedicated to serving just those residents within a specific facility would no longer be valid. Local public health agencies have had agreements with County Boards for closed PODs for those agencies serving persons with developmental disabilities. The challenge now is to create relationships with those new or expanded providers' of adult day services.

To train public health staff and MRC volunteers, the West Central Ohio MRC units comprised of Darke, Shelby, Miami, Champaign, Clark, Preble, Montgomery, and Greene Counties conducted a Point of Distribution (POD) Exercise at the National Center for Medical Readiness on November 7, 2015 using a comparative study design of persons with disabilities and persons without disabilities.

\section{Methods}

The scenario consisted of two cohorts potentially exposed to terrorism related inhalation anthrax exposure. Disaster preparedness, PODs, SNS, and bioterrorism training was followed by a fullscale exercise with volunteers from MRC, Citizen Corps, and Ohio Military Reserve. Volunteers were assigned as POD Staff or Recipients. Staff triaged Recipients for medical prophylaxis (dispensing of ciprofloxacin or doxycycline props) for inhalation anthrax. Time metrics were measured to calculate throughput and service times. The first cohort was all Special Needs (SN) and the second cohort represented the General Population (GP). This study was not submitted to an Institutional Review Board, as it was a public health agency training event to prepare for a public health emergency and to educate disaster responders. This training was consistent with the Core Competencies and Sub competencies for Disaster Medicine and Public Health [19]. Specifically, the ability to describe the potential impact of a mass casualty incident on access to and availability of clinical and public health resources in a disaster or public health emergency.

A traditional medical dispensing clinic was set up to provide medicine to persons potentially exposed to terrorism related inhalation anthrax. Two cohorts (special needs versus general population) were separately evaluated and treated. Volunteers were assigned as POD Staff or Recipients. Staff triaged recipients for medical prophylaxis (dosing with ciprofloxacin or doxycycline) for inhalation anthrax. Time metrics were measured to calculate throughput and service times. Statistical analysis included:

- Critical path method—detecting the stations where a delay could most likely occur.

- General linear modeling of services times.

- Univariate analysis of variance using factors for Clinic Stations and Special Needs. 
General Population

- $59 \%$ female

- $100 \%$ English speaking

- $7 \%$ minors

- $3 \%$ elderly

- $7 \%$ upset

- $3 \%$ wheelchair

- $28 \%$ household medication pickup

- $17 \%$ any other minors

- $10 \%$ allergies

- $21 \%$ medical issues
Special Needs

- $58 \%$ female

- $94 \%$ English speaking

- $13 \%$ minors

- $19 \%$ elderly

- $19 \%$ upset

- $14 \%$ wheelchair

- $87 \%$ household medication pickup

- $55 \%$ any other minors

- $29 \%$ allergies

- $74 \%$ medical issues

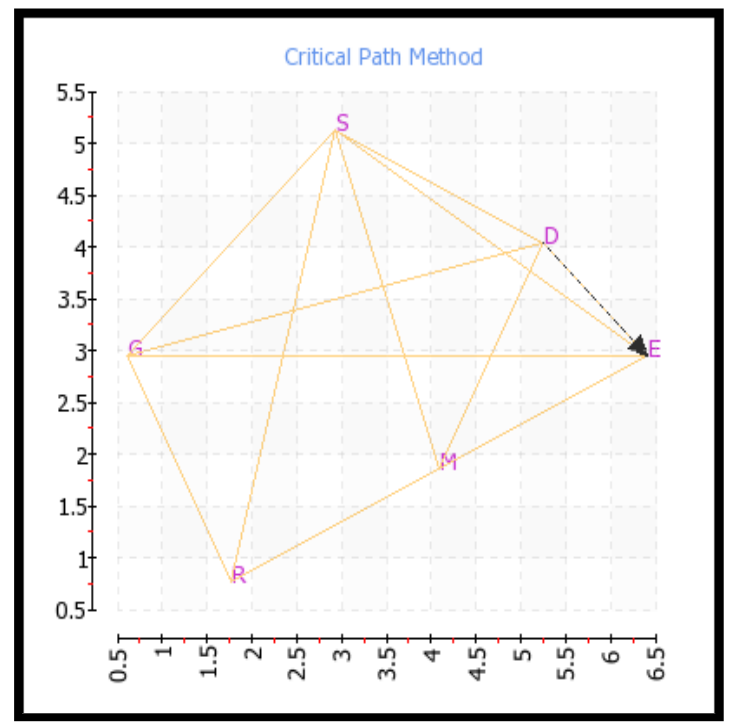

Figure: The dashed line is the critical path. The critical station was Dispensing in both cohorts. $\mathrm{G}=$ Greeter, $\mathrm{R}=$ Registration, $\mathrm{S}=$ Screening, $\mathrm{M}=$ Medical, $\mathrm{D}=$ Dispensing, $\mathrm{E}=$ Exit

\section{Results}

The total time to complete the SN cohort $(n=30)$ was 38 minutes and was 36 minutes for the GP cohort $(n=31)$. Net Total Service Time was nearly double in the SN versus the GP cohort (123 minutes versus 69 minutes). Express Dispensing was less than half the time than for SN versus GP due to less demand on the station (11:22). In a traditional POD setup, Screening, and Medical station resources commensurate with the prevalence of SN are required to meet the needs during community mass care events. The service time for those with Special Needs was 3.73 minutes versus 2.43 minutes for those without $(\mathrm{p}=.082)$. In the general population, service time was 2.48 minutes versus over 4.1 minutes for special needs ( $p=.057)$. Critical Path analysis showed that the Dispensing station was key in either cohorts of special needs or general population. Modeling of wait times showed that children increased wait times by 4.03 minutes ( $\mathrm{p}=.007)$. The critical station was Dispensing in both cohorts. Special Needs cohort could take up to 9 times longer than the general population cohort. Every station could be impacted. Having children increased the wait times by over 4.03 minutes.

\section{Best Practices}

-Standardized format.

-Well marked stations.

-Pre-briefing of staff/volunteers.

-Detailed job descriptions.

-Set objectives.

-Common Language.

-Runners at stations.

-Surge capacity to relieve bottlenecks.

-Flexibility_change as needed. 


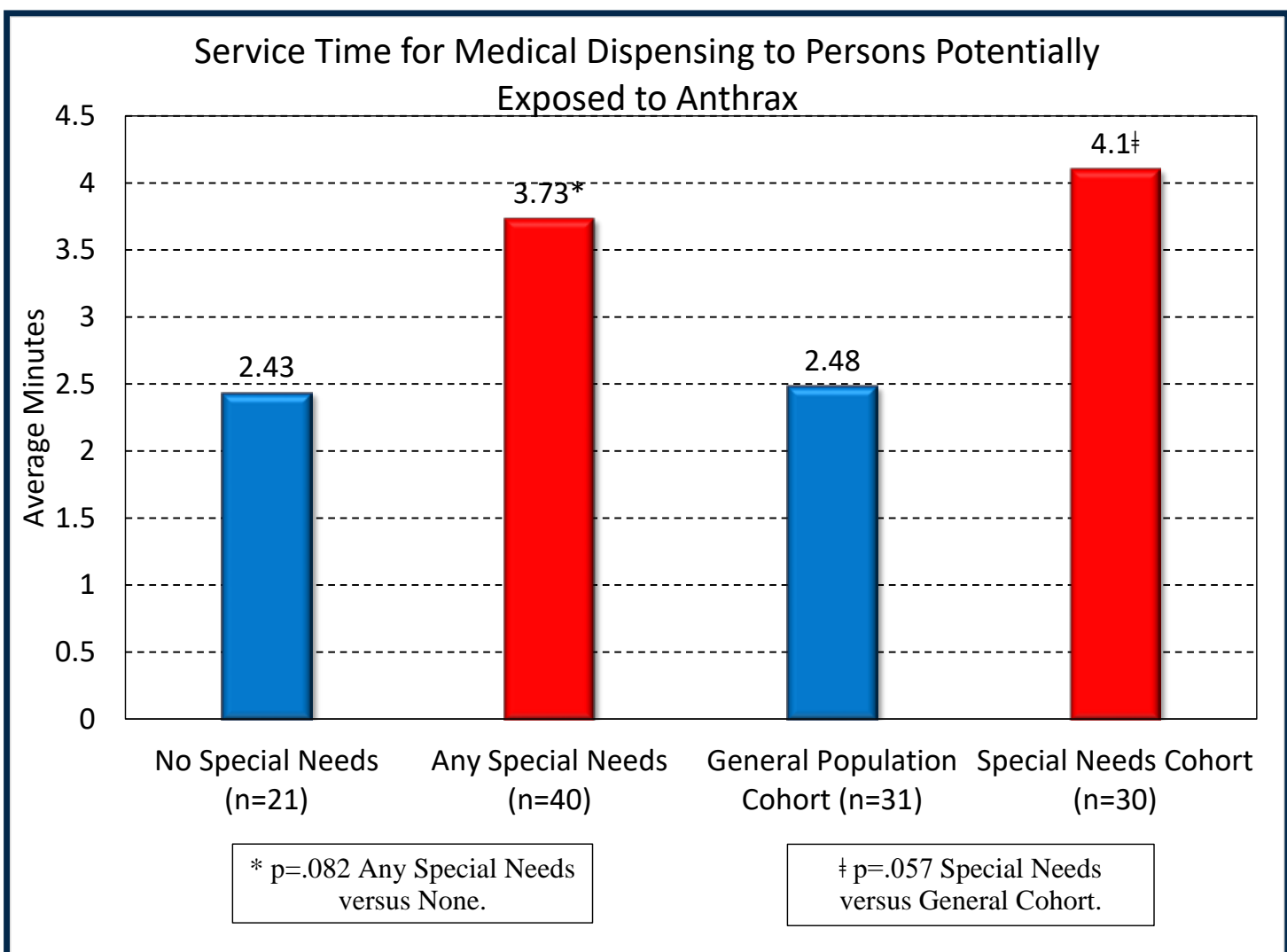

Figure. Comparison of average service times to dispense medicine to persons potentially exposed to anthrax spores by Special Needs status.

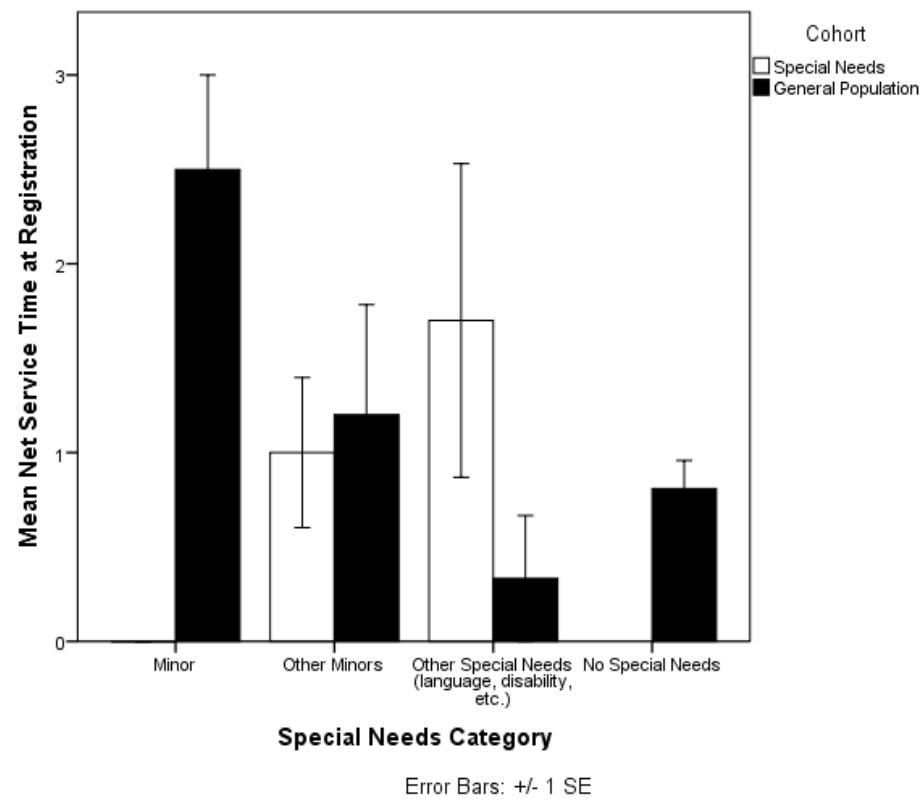

Figure. Comparison of average net service times (excludes in line waiting) at the Registration Station of a Medical Dispensing clinic for persons potentially exposed to anthrax spores. 


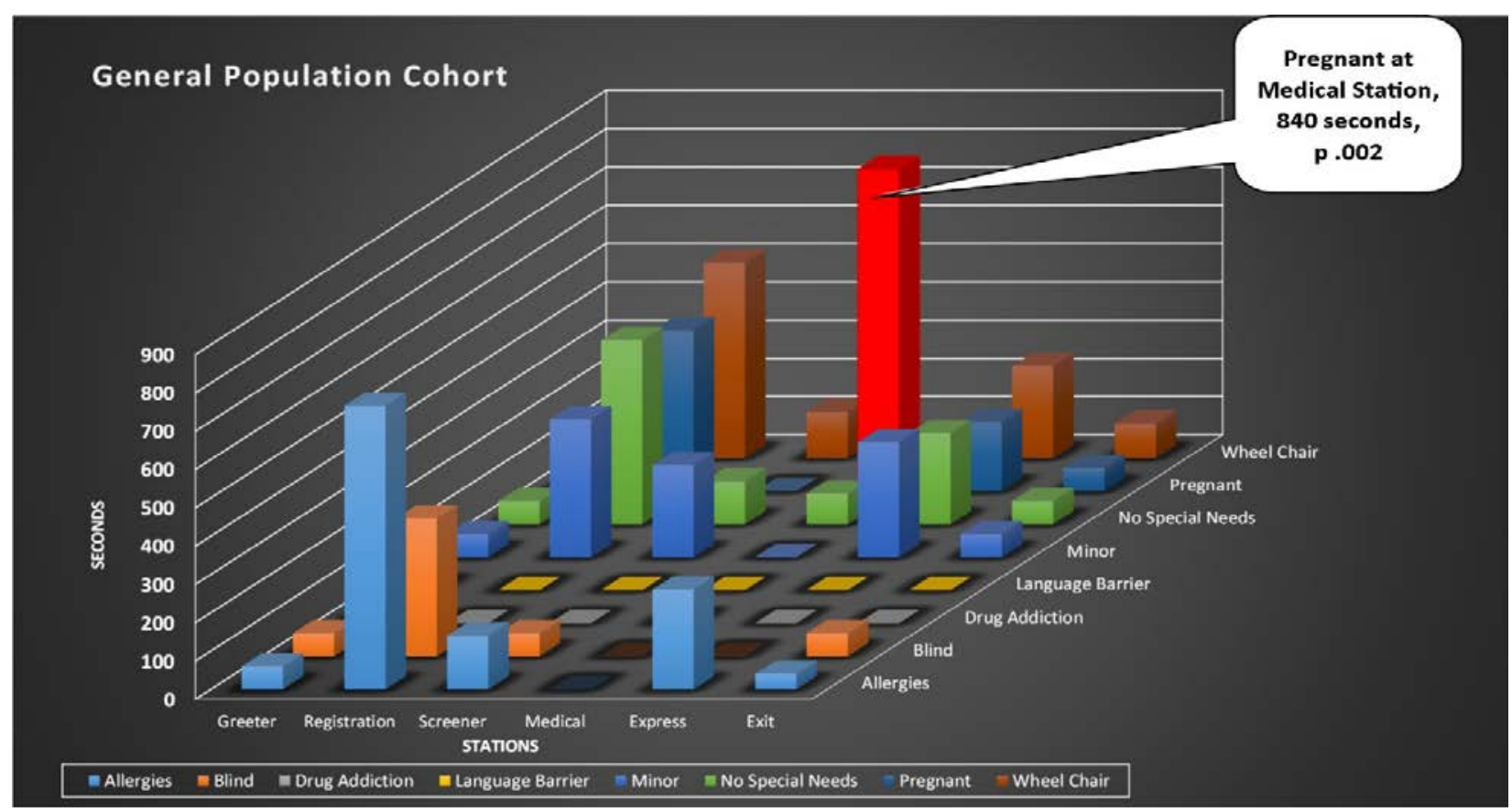

\begin{tabular}{|l|c|c|c|c|c|c|}
\hline Station & Greeter & Registration & Screener & Medical & Express & Exit \\
\hline Allergies & 60 & 740 & 140 & 0 & 260 & 40 \\
\hline Blind & 60 & 360 & 60 & 0 & 0 & 60 \\
\hline Drug Addiction & & & & & & \\
\hline Language Barrier & & & & & & \\
\hline Minor & 60 & 360 & 240 & 0 & 300 & 60 \\
\hline No Special Needs & 60 & 480 & 112 & 79 & 237 & 60 \\
\hline Pregnant & 60 & 420 & 0 & 840 & 180 & 60 \\
\hline Wheel Chair & 60 & 510 & 120 & 0 & 240 & 90 \\
\hline
\end{tabular}

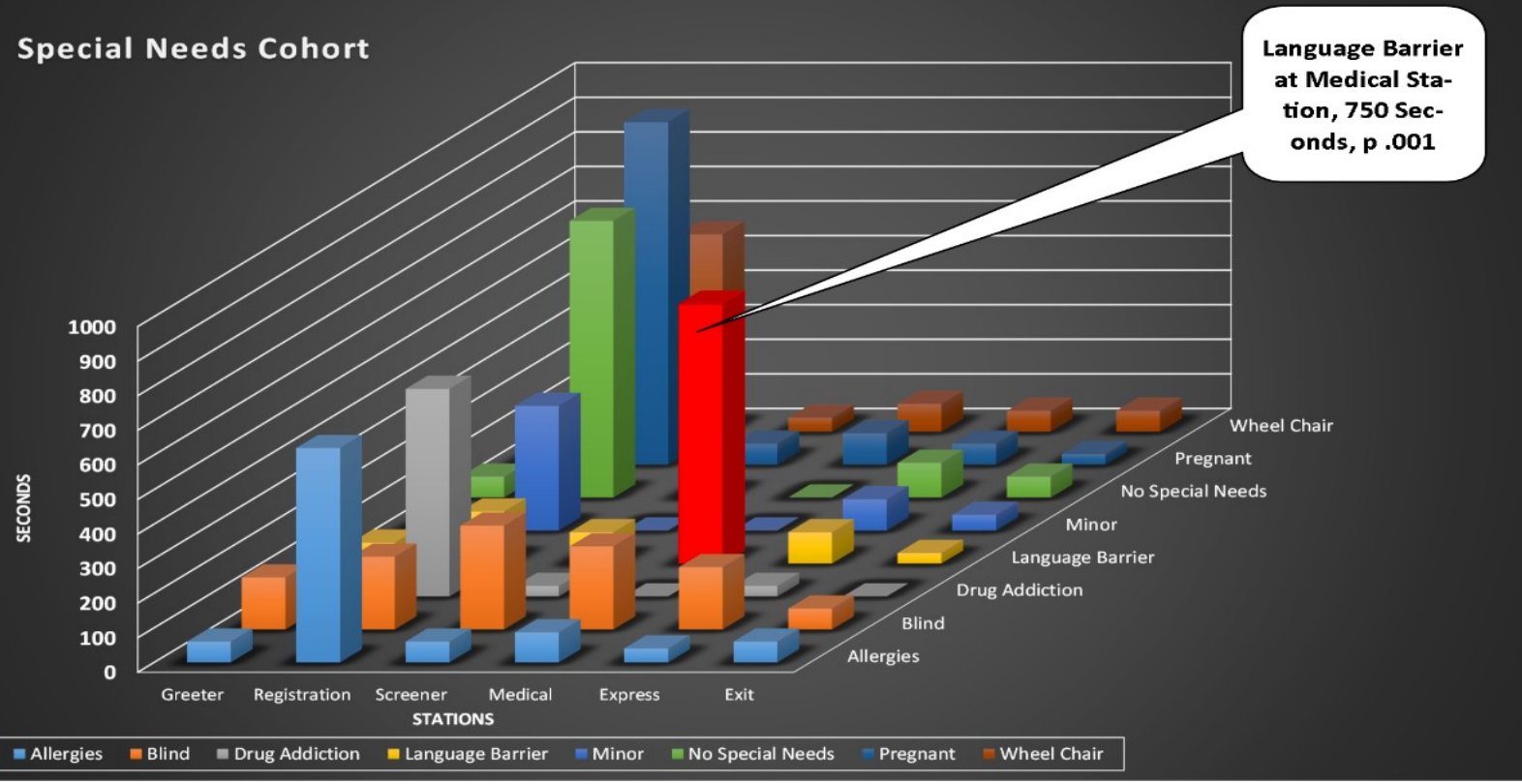




\begin{tabular}{|l|c|c|c|c|c|c|}
\hline Station & Greeter & Registration & Screener & Medical & Express & Exit \\
\hline Allergies & 60 & 620 & 60 & 87 & 40 & 60 \\
\hline Blind & 150 & 210 & 300 & 240 & 180 & 60 \\
\hline Drug Addiction & 60 & 600 & 30 & 0 & 30 & 0 \\
\hline Language Barrier & 60 & 150 & 90 & 750 & 90 & 30 \\
\hline Minor & 195 & 360 & 0 & 0 & 90 & 45 \\
\hline No Special Needs & 60 & 800 & 260 & 0 & 100 & 60 \\
\hline Pregnant & 60 & 990 & 60 & 90 & 60 & 30 \\
\hline Wheel Chair & 90 & 570 & 40 & 80 & 60 & 60 \\
\hline
\end{tabular}

Figure and Data Table: Average Time per Station by Cohort Type.

The subgroup within the general population cohort that had minor children had significantly longer average registration times than the subgroups that had persons with other special needs ( $p=.006$ versus Other Special Needs) or no special needs $(p=.008$ versus No Special Needs). Modeling of service times per station and cohort type found significant delays at the medical station among persons in the general population who are pregnant (840 seconds or 14 minutes, $\mathrm{p}=.002$ ) and persons in the special needs cohort with a language barrier (750 seconds or 12.5 minutes, $\mathrm{p}=.001$ ).

Table 2. Regression Model of Waiting Line Study of Medical Dispensing to Two Cohorts (General Population and Special Needs).

\section{Unstandardized Coefficients \\ Standardized \\ Coefficients}

Sig.

Std.

Model B Error Beta

\begin{tabular}{|c|c|c|c|c|c|}
\hline (Constant) & 660.42 & 63.3 & & 10.43 & 0.000 \\
\hline Screener ILW & 106.01 & 31.6 & 0.343 & 3.36 & 0.001 \\
\hline Medical ILW & 58.72 & 11.1 & 0.516 & 5.3 & 0.000 \\
\hline Express Dispensing ILW & 47.30 & 21.1 & 0.234 & 2.25 & 0.029 \\
\hline $\begin{array}{l}\text { any other minors (if } \\
\text { pickup total }>1 \text { ) }\end{array}$ & 242.65 & 86.8 & 0.275 & 2.8 & 0.007 \\
\hline
\end{tabular}

Note: ILW = In Line Waiting.

Take Home Messages

- Persons with greater medical needs will take longer to process. Expect and plan for more resources to quickly and easily deal with these greater demands.

- Attack bottlenecks early. Formalize adaptability: Set aside a strike team to relieve bottlenecks early.

- More medical staff members are needed to deal with persons with disability.

- Having persons pre-registered would bypass holdups at registration. 
- Having rules to handle the more common medical situations could allow screeners to route persons directly to dispensing.

- The net service times were the same for special needs including wheel chair bound, elderly, and blind persons as for non-special needs. The wait times were longer if children were with the client.

\section{Key Findings}

- Persons with Special Needs required 3.73 to service compared to 2.43 minutes for persons without any special needs $(\mathrm{p}=.082)$.

- The Net Total Service Time was 123 minutes $(n=30)$ for the Special Needs cohort compared to 69 minutes for the General Population cohort.

- Critical Path analysis showed that the Dispensing Station was key in either cohorts of special needs or general population.

- Modeling of wait times showed that children increased wait times by 4.03 minutes $(\mathrm{p}=.007)$.

- In line waiting was increased at the screening (1.76 minutes, p .001), medical (0.98 minutes, $\mathrm{p}<.001)$, and dispensing (0.78 minutes, $\mathrm{p} .029)$ stations.

- Modeling of service times per station and cohort type found significant delays at the medical station among 1) pregnancy (14 minutes or 840 seconds, $\mathrm{p}=.002$ ) in the general population and 2) language (12.5 minutes or 750 seconds, $p=.001$ ) in the special needs cohort.

\section{Service Tips}

- Use standardized formats such as well-marked stations, common terms, pre-briefing of staff and volunteers, detailed job descriptions, and set objectives.

- Attack bottlenecks early. Formalize adaptability: Set aside a strike team to relieve bottlenecks early; Runners at stations; Surge capacity to relieve bottlenecks; Be flexible.

- Persons with greater medical needs will take longer to process. Expect and plan for more resources to quickly and easily deal with these greater demands. Having rules to handle the more common medical situations could allow screeners to route persons directly to overflow stations. For example, pregnancy and language caused significant delays at the stations. Set aside extra staff at overflow stations to handle children, pregnant, and language barriers.

- The wait times were longer at the registration line if children were present. Having persons pre-registered, would bypass holdups at registration.

- The net service times were the same for special needs including wheel chair bound, elderly, and blind persons as for non-special needs, but the in-line waiting (getting to stations) were longer.

- The Dispensing Station is a critical node in the clinic pathway. Have more dispensing staff. 


\section{Strengths}

The volunteers were trained in the morning and they could set up and run the clinic effectively afterwards. The estimates of effect are conservative as the median values of the processing times were used rather than mean for the comparison of service times for modeling except the univariate modeling which used the actual measures.

\section{Discussion}

How does this study relate to public health informatics? Scientists from MIDAS (Models of Infectious Disease Agent Study) funded by the National Institutes of Health model how infectious diseases may emerge and spread, using computers, in order to help public health officials prepare for actual outbreaks [20]. Modeling the response to infectious diseases, especially with the rise of antimicrobial resistance and the potential for bioterrorism, is as important as modeling the infectious diseases. Improving community mass care including mass public health clinics, open PODS to dispense therapies and specific outreach activities to targeted, closed populations with the use of points of dispensing sites (closed PODS) works even in the face of antimicrobial resistance, if the intervention includes triage to isolation and/or quarantine. Likewise, the results of this study processed public health data from the closed POD on two groups of individuals and applied the results to improve the public health response at distributing antibiotics, referring ill or untreatable persons to medical care, isolation or quarantine.

Planned Federal updates to the 2017 Health Care Preparedness and Response Capabilities are focusing on how to assess community planning for children, pregnant women, seniors, and individuals with access and functional needs, including people with disabilities and others with unique needs.

Planning to provide assistance for those individuals who may require additional help before, during, and after an emergency is needed. Specifically, public health planners should be aware of how to provide assistance to people with disabilities and to develop or augment existing response plans for these populations. This support of the health care system to disabled persons would prevent stress on hospitals during an emergency and would allow disabled persons to remain in their residences. Further assessment in and around specific scenarios would be required to assess transport needs to prophylaxis sites, designation of medical care sites for those needing further evaluation, and assessment of specific treatment and access to care needs (e.g., partnering with regional dialysis networks to ensure integration with prophylaxis of disabled persons potentially exposed to anthrax.

The results of the medical dispensing to persons with disabilities were surprising. The service times at the dispensing station were not statistically different between the general population cohort and those with any special needs. However, the time in between stations was increased resulting in total service time for the SN cohort taking longer overall. It is of interest that the presence of children increased the service times. 
Table 3. Numbers of Medicare beneficiaries by the West Central Ohio counties and the number that are electricity dependent. (Sources: U.S. DHHS, August 2016; U.S. Census, July 2012).

\begin{tabular}{lcccc}
\hline County & $\begin{array}{c}\text { Medicare } \\
\text { Beneficiaries }\end{array}$ & $\begin{array}{c}\text { Electricity- } \\
\text { Dependent }\end{array}$ & $\begin{array}{c}\text { Electricity } \\
\text { Dependent } \\
\text { \% }\end{array}$ & Population \\
\hline Champaign & 7,143 & 451 & 1.14 & 39565 \\
\hline Clark & 27,154 & 1,877 & 1.37 & 137206 \\
\hline Clinton & 8,501 & 549 & 1.31 & 41886 \\
\hline Darke & 9,929 & 669 & 1.27 & 52507 \\
\hline Greene & 27,633 & 1,571 & 0.96 & 163587 \\
\hline Miami & 20,070 & 1,170 & 1.13 & 103060 \\
\hline Montgomery & 97,830 & 5,285 & 0.99 & 534325 \\
\hline Preble & 7,994 & 483 & 1.15 & 41886 \\
\hline Shelby & 7,949 & 487 & 0.99 & 49167 \\
\hline
\end{tabular}

Note: The Medicare data is from the U.S. Department of Health \& Human Services and shows the number of Medicare (over 2.4 million in the U.S.) beneficiaries rely upon electricity-dependent medical and assistive equipment, such as ventilators and wheel chairs and is updated monthly [21].

Given that just in time training was provided, concerns about volunteers and local public health staff having the capability to conduct a mass-dispensing clinic are unfounded, as the volunteers and limited staff were effective in providing medicine to persons exposed to inhalation anthrax regardless of their special needs status. Additional resources should be put in place for preregistration or additional onsite registration, especially for those with children. Mass prophylaxis dispensing clinics should anticipate significant delays in service times for persons with special needs including pregnant, language barriers, and children. Clinics dispensing to cohorts composed entirely of persons with special needs should reorder their traditional single waiting line per station to having an 'inline' medical exam station prior to the dispensing station (and an overflow medical exam station for pregnant and persons with language barriers).

\section{Recommendations}

Ensure memorandums of understanding are in place for select agencies involved in providing services to those with special needs to allow for public health to provide for closed POD dispensing to their clients. The critical node in a traditional clinic pathway is the dispensing station, therefore it is imperative to have a sufficient number of medicine dispensers to avoid a delay in service times. If a closed POD is not an alternative strategy to a traditional dispensing clinic, service times should be expected to be twice as long. Assign extra capacity at the medical station area for special needs involving children, language, or pregnancy issues. 


\section{Limitations}

Real world events may differ significantly. There are the typical concerns about security and logistics that vary with each clinic location. The two critical differences that will inevitability vary regardless of the clinic location, is the amount of exposure and the susceptibility of those exposed. The incubation period can be quite different depending on the amount of spores inhaled with the median incubation period being 9 days (95\% confidence interval 8 to 10) and possibly less than 2.5 days with higher doses [22]. This is important because with shorter incubation, prophylaxis to be implemented very quickly if lives were to be saved. Those with special needs will on average have more vulnerabilities and will require prophylaxis to be dispensed in less time after exposure to increase the rate of survival. When modeling using station and cohort type as factors small numbers caused great variability in standard errors possibly missing important causes of delays. More research is needed to explore dispensing to persons with drug addiction, blind persons, and other segments where this evaluation had great delays but non-significant results.

\section{Acknowledgements}

The authors would like to thank the Public Health, Medical Reserve Corps and Ohio Military Reserve volunteers who participated on November 7, 2015 in staffing the stations and the staff at the Wright State University's National Center for Medical Readiness.

\section{References}

1. Brannen DE, Stanley SA. 2004. Critical issues in bioterrorism preparedness: before and after September 2001. J Public Health Manag Pract. 10(4), 290-98.

2. Kaplan EH, Craft DL, Wein LM. 2002. Emergency response to a smallpox attack: the case for mass vaccination. Proc Natl Acad Sci USA. 99(16), 10935-40. Epub Jul 2002.

3. Davis TC, Fredrickson DD, Kennen EM, Arnold C, Shoup E, et al. 2004. Childhood vaccine risk/benefit communication among public health clinics: a time-motion study. Public Health Nurs. 21(3), 228-36.

4. Kaplan EH, Craft DL, Wein LM. 2003. Analyzing bioterror response logistics: the case of smallpox. Math Biosci. 185(1), 33-72.

5. Washington ML, Mason J, Meltzer MI. 2005. Maxi-Vac: planning mass smallpox vaccination clinics. J Public Health Manag Pract. 11(6), 542-49.

6. Brannen DE, McDonnell M, Howell M, Sesler S, Jez S, et al. (2006). Cost analysis and critical path method of multiple mass dispensation clinics in Greene, Preble, Montgomery, Clark, and Miami Counties. Ohio Public Health Epidemiology Conference. August 1, 2006.

7. Brannen DE, McDonnell MA, \& Schmitt, A. (2013). Organizational culture on community health outcomes after the 2009 H1N1 pandemic. Journal of Organizational Culture Communications \& Conflict, (17)1. 
8. Greenfieldboyce, Nell. Inside A Secret Government Warehouse Prepped For Health Catastrophes. Morning Edition. June 27, 2016 4:56 AM ET

9. Bower WA, Hendricks K, Pillai S, Guarnizo J, Meaney-Delman D, \& Centers for Disease Control and Prevention (CDC). 2015. Clinical Framework and Medical Countermeasure Use During an Anthrax Mass-Casualty Incident. MMWR Recomm Rep. 64(4), 1-22.

10. Hearne SA, Davis M, Segal LM, Unruh PJ, Earls MJ, et al. 2004. Trust for America's Health. Ready or not? Protecting the public's health in the age of bioterrorism. Biosecur Bioterror. 2(1), 47-50.

11. Levi J, Segal LM, Liebermann DA, May K, Lang A, et al. (2012 December). Ready or Not? Protecting the Public from Diseases, Disasters, and Bioterrorism. December 2012. Issue Report. Trust for America's Health. www.healthyamericans.org.

12. Ludtke JR, Narayan R, Matariyeh A, Brannen D, Caudill K, et al. 2014. Willingness to respond for radiologic incidents: a hands-on approach. Am J Disaster Med. 9(4), 259-72. doi:10.5055/ajdm.2014.0178.

13. Brannen DE, Barcus R, McDonnell MA, Price A, Alsept C, et al. 2013. Mental health triage tools for medically cleared disaster survivors: an evaluation by MRC volunteers and public health workers. Disaster Med Public Health Prep. 7(1), 20-28.

14. Brannen DE, Schmitt A, McDonnell M. 2011. Critical Issues Faced by MRC in a Special Needs Shelter. Domestic Preparedness Journal. 7(5), 17-21.

15. Fannin A, Brannen DE, Howell M, Martin S. 2015. Using Functional Needs and Personal Care Assistance Rather Than Disability Status During Chronic Care Triage in Community Mass Care. Disaster Med Public Health Prep. 9(3), 265-74. Epub Mar 2015. doi:http://dx.doi.org/10.1017/dmp.2015.21.

16. Waldrop J, Stern SM. Disability Status 2000: Census 2000 Brief. March 2003. http://www.census.gov/prod/2003pubs/c2kbr-17.

17. Public Health - Dayton and Montgomery County. Mass Dispensing and Vaccination Plan. 2011 May 14: 6-7.

18. Kenny J. Center for Medicaid and Medicare (CMS) mandated that all County Boards of Developmental Disabilities cease Adult Day Services by 2019. WYSO Weekend, Jun 27, 2016. WYSO Public Radio.

19. Walsh L1. 2012. Subbarao I, Gebbie K, Schor KW, Lyznicki J, Strauss-Riggs K, Cooper A, Hsu EB, King RV, Mitas JA 2nd, Hick J, Zukowski R, Altman BA, Steinbrecher RA, James JJ. Core competencies for disaster medicine and public health. Disaster Med Public Health Prep. 6(1), 44-52. doi:10.1001/dmp.2012.4.

20. Modeling Infectious Diseases Fact Sheet - National Institute of General Medical Sciences [Internet]. U.S National Library of Medicine. U.S. National Library of Medicine; [cited 
2016Oct3]. Available from:

https://www.nigms.nih.gov/research/specificareas/midas/background/pages/factsheet.aspx

21. Pages - default [Internet]. Pages - default. [cited 20160ct3]. Available from: http://www.phe.gov/empowermap/pages/default.aspx

22. Wilkening DA. 2008. Modeling the incubation period of inhalational anthrax. Med Decis Making. 28(4), 593-605. Epub Jun 2008. doi:http://dx.doi.org/10.1177/0272989X08315245. 